\title{
Probabilistic design and analysis of building performances: methodology and application example
}

\author{
Liesje Van Gelder*, Hans Janssen, Staf Roels \\ Building Physics Section, Department of Civil Engineering, KU Leuven, Kasteelpark Arenberg 40 Bus 2447, 3001 Heverlee, Belgium
}

\begin{abstract}
Both building performance analysis and multi-criteria performance optimisation often use deterministic simulations. Since many influencing parameters are generally inherently uncertain, this may lead to unreliable predictions of design impact. Therefore, this paper proposes a probabilistic analysis and design method to incorporate these uncertainties. The embedded Monte Carlo based uncertainty and sensitivity analyses investigate the output distributions. To greatly reduce computational efforts, meta-models can be incorporated, replacing the original model. Additionally, multi-layered sampling schemes are used to subject all design options to the same uncertainties and to check the validity of optimisation results for potential scenarios. Since reliability is a key aspect in this methodology, the paper also focuses on output convergence and method reliability.

To optimise both average performances and spread, effectiveness $\varepsilon$ and robustness $R_{P}$ are introduced as output uncertainty indicators, inspired by robust design. Here, effectiveness is defined as the ability of the design option to optimise the performance, while robustness is defined as the ability to stabilise this performance for the entire range of input uncertainties.
\end{abstract}

The successive methodology steps are explained using a simplified application example.

Keywords:

probabilistic analysis, probabilistic design, robust design, optimisation, Monte Carlo

\section{Introduction}

Building performance analysis and design targets the examination and optimisation of the building performances. Analyses typically assess performances of a known construction for comparison with performance criteria, while designs do the same for a number of potential solutions in a search for the optimal solution. For example, the analysis of Abuku et al. [1] examines the impact of wind-driven rain on mould growth risk, indoor climate and energy consumption in historic brick buildings, while the design of Verbeeck and Hens [2] minimises energy use, environmental impact and financial costs for extremely low-energy dwellings.

Both studies, and many others with them, make use of deterministic simulations, hence neglecting the inherent variability and uncertainty of geometries and configurations, of material and component properties, of internal loads and boundary conditions, of economical and environmental parameters, ... A deterministic approach may hence lead to inconclusive analyses and non-optimal designs, and a probabilistic methodology is therefore to be

\footnotetext{
*Corresponding author

Email address: liesje.vangelder@bwk.kuleuven.be (Liesje Van Gelder)
}

preferred. And while a lot of work has already been done with respect to probabilistic analysis and design in building performance assessment, much of this has focused on specific and delimited issues. The current state-of-theart thus remains highly fragmented. This article therefore aims at merging that fragmented knowledge into a structured and comprehensive methodology for probabilistic analysis and design in building performance assessment.

Section 2 explains the background and objectives of this paper. The global methodology is introduced in section 3 and extensively explained and illustrated in section 5. As an example, the net present cost of a low-energy dwelling is optimised for several scenarios to obtain a robust costeffective dwelling. This case study is described in section 4 . Finally, section 6 discusses further research opportunities.

\section{Background and objectives}

The methodology for probabilistic analysis and design in this paper is built on several state-of-the-art principles to calculate and analyse the output uncertainty. Each of these principles is briefly explained in this section.

\subsection{Monte-Carlo-based techniques}

Probabilistic procedures have been introduced into building physics over the last decades, often inspired by 
other engineering disciplines [3]. Two fundamental aspects are uncertainty quantification and sensitivity analysis, to transform the variabilities of the input to the uncertainties of the output and to identify these input parameters that are most dominant in this transformation. Due to the complex, non-linear and transient character of most building performance problems, Monte-Carlo-based techniques are often preferred for these goals.

Since the first steps of Lomas and Eppel [4] and Macdonald and Strachan [5] in probabilistic analysis in thermal building simulation, a huge progress has already been made in terms of time efficiency. Output convergence and sampling efficiency have been studied, resulting in quasi-Monte Carlo sampling schemes instead of random sampling as generally described by Janssen [6] and illustrated by Burhenne et al. [7]. Furthermore, Eisenhower et al. [8] introduced the use of meta-models into building energy optimisation. Meta-modelling allows replacing a time-inefficient model by a model with a highly reduced calculation time.

However, in the probabilistic studies mentioned above, the input parameter variations were often arbitrarily chosen, what may of course influence the output uncertainty as well. Selecting the most dominant parameters with sensitivity analysis, eases collecting the input data. If a sufficient number of measurements is available, the input distributions of these parameters can be determined based on Bayesian calibration $[9,10]$. Unfortunately, this is usually not the case. Most dominant parameter distributions are thus usually chosen as accurately as possible.

\subsection{Probabilistic output analysis}

As a consequence of the above-mentioned probabilistic procedures, the resulting building performance indicators in probabilistic analysis or design are no longer singlevalued, but are to be evaluated based on their probability density distribution. Booth and Choudhary [10] visually compared output distributions for some refurbishment measures in order to decide how to cost-effectively retrofit the UK housing stock. Although they describe the overall methodology extensively, the question how probabilistic output distributions should be numerically evaluated and optimised in probabilistic design is not answered.

This in turn introduced considerations of robustness into building performance design, to allow not only an assessment of the mean performance but also of its possible spread around that mean. In robust design, mean performance is indeed optimised while spread is minimised [11], resulting in a design that can resist the influence of uncontrollable factors as good as possible [12]. In previous studies such as Hoes et al. [13], robustness was however only examined after optimal design solutions were selected, partially missing the benefits.

\subsection{Paper objectives}

Literature shows that much effort has been put in efficiently calculating output uncertainties in probabilistic analysis and some in using these uncertainties for decision making in probabilistic design; however, this is still highly fragmented. Taking into account the described achievements and shortcomings, this paper proposes an efficient way to combine and improve these elements in an overall applicable, structured and comprehensive methodology for probabilistic analysis and design to quantify performance spread and to compare several design options.

First of all, all design options are combined with the same uncertain parameter values, as is done in robust design [14] and for example also by Booth and Choudhary [10]. Furthermore, in many analyses or designs the explicit evaluation of the result for different scenarios - be they defined based on user behaviour, economic parameters, climate change, ... - may be wanted as suggested by Hopfe and Hensen [15]. This asks for the formulation and application of Monte Carlo multi-layered uncertainty schemes.

To enable numerical evaluation of these schemes, effectiveness and robustness indicators are proposed, inspired by robust design. Effectiveness is defined as the ability of the design option to optimise the performance, while robustness is defined as the ability to stabilise this performance for the entire range of input uncertainties.

As the proposed methodology may rapidly result in a high computational cost, even for very time-efficient models as those in referred literature, it is preferable to replace the original model with a simpler and much faster metamodel [8]. Furthermore, both convergence and sampling efficiency are crucial to overcome time issues while obtaining reliable results [6], as well as sensitivity analysis to select and determine input parameter distributions based on measurements or expertise [10].

In literature, probabilistic design seems to be more challenging and applications are less widespread. The major advance of the methodology proposed in this paper is thus in terms of probabilistic design, whereas probabilistic analysis is seen as a special application.

\section{Global methodology}

To reliably incorporate uncertainties in all aspects of performance analysis and design, this paper proposes a comprehensive probabilistic methodology, of which the main ideas were introduced in section 2.3. Four main steps can be distinguished (see Fig. 1), of which only the main aspects are discussed here, while they are explained and illustrated in more detail in the referred subsections of section 5. Following some ideas on robustness from manufacturing design in the output evaluation, effectiveness and robustness indicators are introduced.

\subsection{Methodology overview}

The probabilistic design methodology consists of four steps (see Fig. 1): preprocessing, preliminary screening, updating and probabilistic design. These steps analogously select the input parameters and distributions (step 


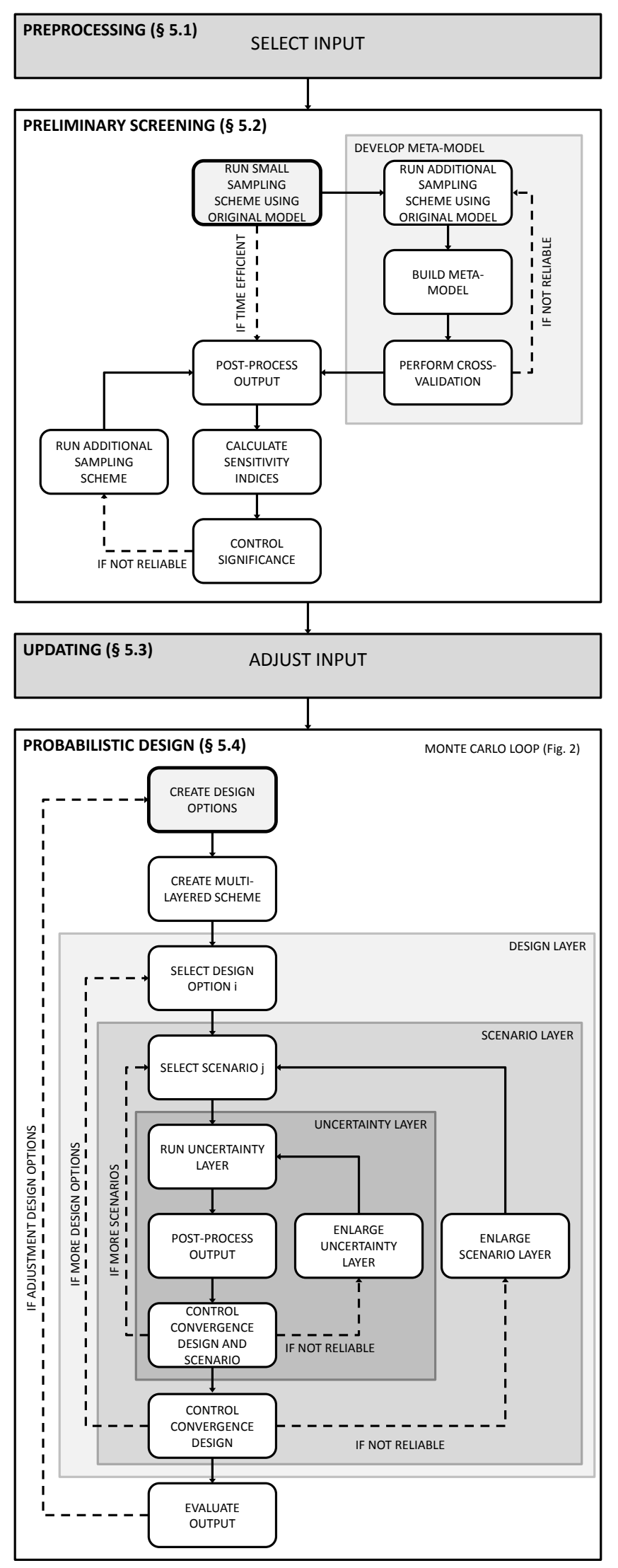

Figure 1: Flowchart probabilistic design.
1), determine the most dominant input parameters and develop a meta-model to improve calculation efficiency (step 2 ), update the input distributions (step 3), and finally perform the actual probabilistic design (step 4).

Contributing input parameters of this probabilistic design can be divided into three categories. Design parameters, such as the preferred air tightness or thermal resistance, are fully controllable. They are the unknown parameters in the design process, but once a design option is selected, the parameter values are known. Inherently uncertain parameters, such as workmanship and user behaviour, are completely uncontrollable by the designer as their values are neither known in the design process nor after, but they can significantly influence the design performance. Finally, scenario parameters are inherently uncertain parameters dealing with potential future scenarios, such as economic or climatic evolutions, for which an explicit evaluation is wanted.

The probabilistic design (step 4) is therefore performed through a Monte Carlo loop with a multi-layered sampling scheme which enables sorting parameters by their conceptual meaning. By ascribing these parameter categories to a different layer in a multi-layered sampling scheme as shown in Fig. 2, all design options are subjected to the same uncertainties and a direct comparison for several future scenarios is enabled. As a result, this probabilistic design can be used as an effective decision tool.
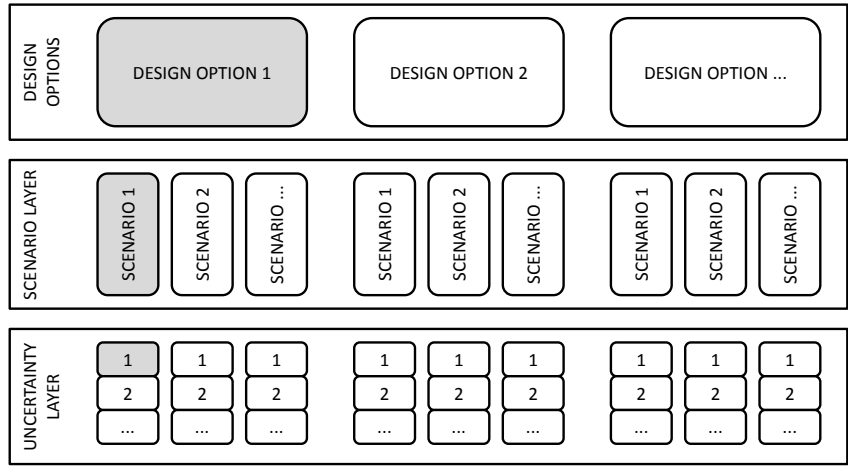

Figure 2: Multi-layered sampling scheme. The first Monte Carlo run is indicated in gray.

Prior to performing such a probabilistic design, the problem is first preprocessed (step 1) to select the output parameters needed for decision making and a suitable simulation model. Both stationary or dynamic, and simplified or complex models can be chosen. Contributing input parameters are determined and fixed values or (provisional) input distributions are ascribed for respectively deterministic and stochastic parameters.

Since the proposed multi-layered sampling scheme significantly increases the needed number of runs, timeinefficient models are preferably replaced by a meta-model in the preliminary screening (step 2). Therefore, training and validation sets are run in the original model to construct and validate the meta-model. Due to the extent of 
the multi-layered scheme, smaller sampling sets are used. These sets are also used to calculate sensitivity indices to rank the input parameters from most to least influencing the output distributions.

Based on this sensitivity ranking, the provisional distributions of most influencing parameters are updated (step 3 ), while the less influencing parameters can be omitted. Limiting the number of parameters eases collecting the required input distributions as this can be time-consuming. Moreover, this improves sampling efficiency and limits the number of considered design options in the multi-layered scheme. This stresses the importance of the preliminary screening in addition to the actual probabilistic design.

Note that in a probabilistic analysis, only one design is investigated, thus this can be examined as a probabilistic design problem with only one considered design option, consisting of one or more design parameters. The execution of the four steps of Fig. 1 is completely analogous.

\subsection{Robust design output evaluation}

To evaluate the output in probabilistic design, building mechanics and building physics $[13,16]$ have already adopted some ideas on robustness from manufacturing design, where an overall good performance and small spread are preferred. However, in these studies robustness was only examined after optimal design solutions were selected, partially missing the benefits. Hopfe et al. [17, 18] for instance, investigated robustness by means of best and worst performances, which is however also a limited approach to robustness. Therefore, to fully exploit the opportunities of the robust design approach, the performance median and spread has to be simultaneously optimised as can be done by standard multi-criteria optimisation methods.

To numerically evaluate the obtained output distributions in such optimisation methods, this paper proposes effectiveness $\varepsilon$ and robustness $R_{P}$ as output uncertainty indicators [19]. For a design option $x_{i}$, which is a set of design parameter values, $\varepsilon$ and $R_{P}$ are formulated as:

$$
\begin{aligned}
& \varepsilon\left(x_{i}\right)=1-\frac{y_{50}\left(x_{i}\right)-y_{\min }}{y_{50}-y_{\min }} \\
& R_{P}\left(x_{i}\right)=1-\frac{y_{50+\mathrm{P} / 2}\left(x_{i}\right)-y_{50-\mathrm{P} / 2}\left(x_{i}\right)}{y_{50+\mathrm{P} / 2}-y_{50-\mathrm{P} / 2}}
\end{aligned}
$$

with $y_{q}$ the $q^{\text {th }}$ percentile under full uncertainty, $y_{q}\left(x_{i}\right)$ the $q^{\text {th }}$ percentile after selecting design option $x_{i}$ and $P$ the user specified percentage of included sample points, as illustrated in Fig. 3. $y_{\text {min }}$ corresponds to the minimal calculated value which is not an outlier, whereby an outlier is defined as a sample point smaller than $y_{25}-1.5\left(y_{75}-y_{25}\right)$. In this definition the performance indicator $y$ is defined in such a way that it is greater or equal to zero and to be minimised. In this paper, we choose $P$ equal to $95 \%$.

Effectiveness thus describes how the deviation between median performance and optimal performance $\left(y_{\min }\right)$ for a design option improves compared to the deviation under

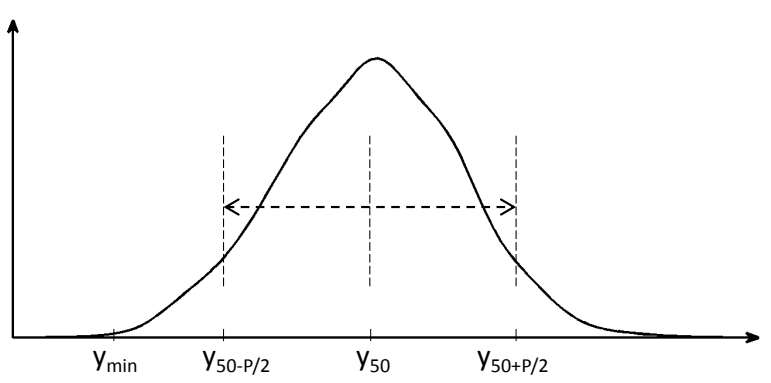

(a) PDF of output parameter value $y$ under full uncertainty of design and uncertain parameters and considered scenarios.

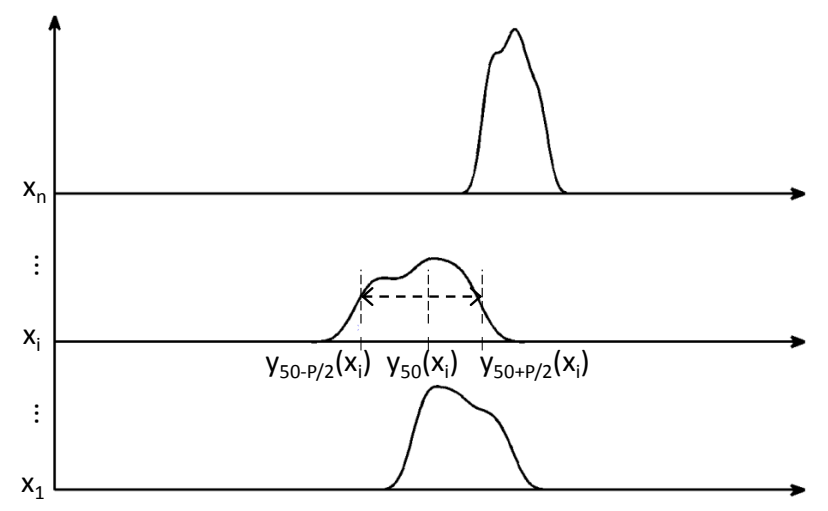

(b) PDF of output parameter value $y$ for design options $x_{i}$ under uncertainty of uncertain parameters and considered scenarios.

Figure 3: Probability density functions (PDF) of output parameter value $y$ under full uncertainty and after selection of design options $x_{i}$.

full uncertainty. The robustness is analogously determined as the improvement the performance spread of a design option makes in proportion to the performance spread under full uncertainty. According to this definition a measure with an effectiveness and robustness of one is the best possible, while negative values are to be avoided. Note that these indicators are depending on the considered scenarios and the set of potential design options. For each scenario, adopted indicators can be analogously calculated. $y_{q}\left(x_{i}\right)$ is then the $q^{\text {th }}$ percentile after selecting design option $x_{i}$ and the considered scenario value.

\section{Case study}

To illustrate the developed methodology, this paper presents a simplified probabilistic design problem of a semi-detached house as shown in Fig. 4. Numerous building physical design options will be compared based on the net present cost as output parameter in order to select the most cost-effective and cost-robust design.

\subsection{Building model}

The building model has a floor area of $140 \mathrm{~m}^{2}$, an uninsulated basement and overhangs for sun shading. Two separate zones are modeled to differentiate between day and night zone. The adjacent dwelling is considered at 


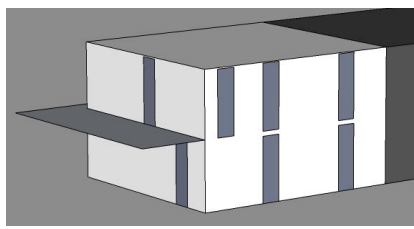

(a) North facade

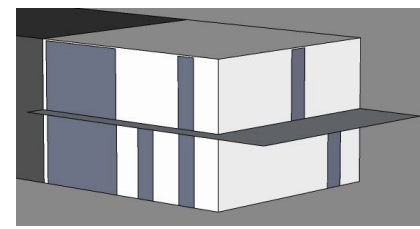

(b) South facade
Figure 4: Case study - semi-detached house

a constant temperature of $19^{\circ} \mathrm{C}$. The heat demand of the dwelling is simulated for a reference year in Uccle, Belgium, using a transient building energy simulation (BES) tool in Modelica [20]. To simulate the heat demand, an ideal heating system is assumed, which is controlled using simplified user and temperature profiles. In summer the heating system is switched off.

\subsection{Model output and post-processing}

The dynamic BES-model output is used to directly calculate the yearly heat demand. This will be post-processed to calculate the net present cost.

In this simplified case study, the net energy demand is derived considering a condensing gas boiler as heating system with a delivery efficiency of $89 \%$ and a generation efficiency of $97 \%$ according to the EU Energy Performance of Buildings Directive EPBD [21] for Flanders, Belgium. This net energy demand is also augmented with the auxiliary energy for ventilation, which is assumed direct current and estimated according to EPBD as well.

Thereafter, this net energy demand is post-processed to compute the associated yearly energy cost. The net present cost is then calculated according to the European standard EN ISO 15459 [22] with a calculation tool developed in the research project IWT TETRA BEP2020 [23]. This standard only takes the cost of energy related components into account. In the current analysis, all costs which are assumed identical for all considered dwelling designs, such as foundation and household electricity, are thus neglected. The heating system cost is also not included as this is, for reasons of simplicity, assumed independent of the heat demand. In the calculations, the lifespan taken is 30 years, the market interest rate $4.5 \%$ [22] and the inflation rate $2.3 \%$ [23]. Inflation is estimated based on Belgian data from the past ten years. The current average energy cost for electricity is $0.21 \mathrm{EUR} / \mathrm{kWh}$ and for gas $0.07 \mathrm{EUR} / \mathrm{kWh}$ and is assumed to increase or decrease every year by the same percentage.

\section{Methodology application}

The methodology introduced in section 3 is explained in more detail in this section and illustrated based on the case study described in section 4 . Fig. 1 gave an overview of the methodology with references to the subsections below describing the four main methodology steps:
1. Preprocessing

2. Preliminary screening

3. Updating

4. Probabilistic design

\subsection{Preprocessing}

First, the output parameters which are needed in the probabilistic design problem, and a suitable model to simulate them, are selected. Successive models can be used as well to calculate derived output parameters. Most deterministic models can be included in a Monte Carlo loop by changing the input values, thus stationary or dynamic and both simplified or very complex models can be chosen. As explained in section 4 for this simplified academic example, a transient BES-model is used to calculate the net heat demand, whereafter the considered output parameter, the net present cost, is computed with a subsequent cost calculation tool.

Then, potentially contributing input parameters can be identified through a qualitative approach exploring the problem. Based on expertise or measurement campaigns one then can determine which parameters are to be considered deterministic and which stochastic. Parameters inherently having a significant uncertainty, such as user behaviour, are obviously stochastic. When it is not sure whether parameter uncertainties will influence the investigated outputs, it is desirable to assume them stochastic, since their impact will be checked anyway in the preliminary screening (in section 5.2). All stochastic input parameters taken into account in the current illustrative case study, are listed in the first column of Table 1, according to the parameter categories described in section 3.1.

Finally, for all considered input parameters, fixed values and probability distributions for respectively deterministic and stochastic parameters need to be assigned as accurately as possible for the considered problem and its context. For example, data for renovation studies might differ from data for new constructions, and may furthermore depend on location, climate and building tradition. Moreover, some parameters can be variable in time and space. This, as well as the fact that certain parameters can be correlated, can be taken into account.

Table 1 shows the ascribed input distributions for the stochastic input parameters of the case study in the second column. Some parameters have strictly discrete values, while others are described by continuous uniform, normal or Weibull distributions. These distributions are all based on the values that can be expected for newly built dwellings in Belgium.

Two ventilation systems are implemented and labeled corresponding to Belgian standard NBN D 50-001 [24]. Type D is a mechanical system, equipped with heat recovery, of which the efficiency is uniformly distributed. Five commercially available window types are considered, indicated with their U-value and the solar energy transmittance of the glass (g-value). 
Table 1: Stochastic design, uncertainty and scenario parameters.

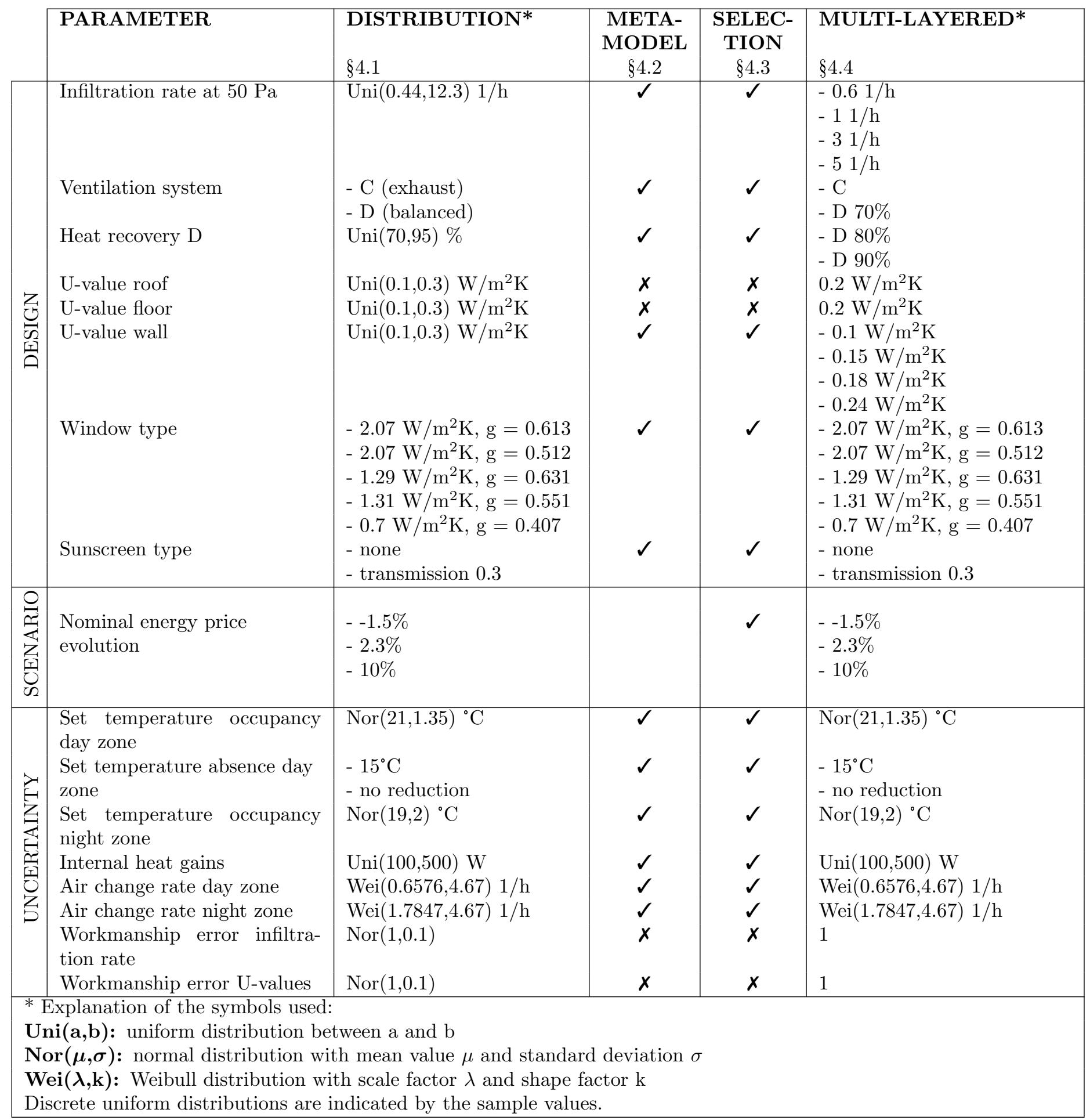

Since user behaviour has a large impact on dwelling energy demand [25], several set temperatures are taken into account of which the distributions are inspired by a measurement campaign of 70 new dwellings is Flanders (Belgium) [26]. Occupancy profiles are considered deterministic to simplify the case study.
Air change rates are zone and building dependent due to nominal ventilation flows and zone volumes. The measurement campaign [26] indicates that indoor air quality is very variable, supposing that in only a part of the dwellings the nominal ventilation rate is reached. Based on these findings, Weibull distributions are proposed. 
Workmanship can influence the performance of air tightness and insulation as well. To account for this, a workmanship error is multiplied with the design values:

$$
X_{\text {res }}=X_{\mathrm{des}} \cdot e_{\mathrm{work}}
$$

with $X_{\text {res }}$ the resulting parameter value, $X_{\text {des }}$ the design value and $e_{\text {work }}$ the workmanship error. These errors are assumed normally distributed with a standard deviation of $10 \%$.

At last, when calculating net present costs and implicitly weighing investment costs against energy costs, future energy prices are assumed of great importance. This inherently uncertain parameter will be placed in the scenario layer to allow for an explicit evaluation. Although price evolutions are difficult to predict, three potential scenarios are assumed. A small drop (-1.5\%), a price evolution equal to inflation $(+2.3 \%)$ and a sharp increase $(+10 \%)$ are considered equally probable. This parameter is obviously not included in the BES-model, but only in the cost calculation tool.

\subsection{Preliminary screening}

To gain some understanding of the variation of the output, a preliminary screening is performed. A sampling scheme is run and sensitivity indices are calculated to determine which input parameters need to be taken into account (section 5.3). For time-inefficient models, a metamodel is constructed to replace the original model.

\subsubsection{Sampling scheme}

Based on previous research on sampling efficiency [6], space-filling sampling is preferred as it has faster convergence than random or Latin Hybercube sampling. In this paper a maximin sampling scheme is applied, a distancebased space-filling scheme that maximises the minimal distance between Latin Hypercube sampling points [27].

In the preliminary screening, all uncertainties are sampled together in a small sampling scheme, and the original model is run to investigate the output uncertainty range that can be expected. In order to reach convergence, gradually more samples can be added. Depending on the sampling strategy, more samples can be added one by one as for Sobol sampling, or new sample sets need to be added as for Latin Hypercube based sampling. These sets can be created by random column permutations of the sampling scheme as the columns refer to the input parameters [6].

In this case study the parameters in Table 1 are sampled 20 times resulting in yearly heat demands between 43 $\mathrm{kWh} / \mathrm{m}^{2}$ and $153 \mathrm{kWh} / \mathrm{m}^{2}$. Column permutations will be used to add sample sets in next subsections.

\subsubsection{Meta-model}

Meta-models, also known as surrogate models, aim at mimicking the original simulation model, but with a highly reduced calculation time, which facilitates the optimisation procedure [8]. To construct such a meta-model, several simulations are run with the original tool. This data will be referred to as training data and are used to build the model. For each output parameter, such as the heat demand in this case study, a meta-model is built separately based on available techniques, such as multivariate adaptive regression splines (MARS) or neural networks. MARS, a regression method that approximates the simulated outputs with hinge functions [28], is used in this paper. To confirm the meta-model's reliability, additional simulations are used as validation data. In general, all input and output data are standardised (zero mean, unit variance) to overcome influences from parameter units. If the original model is already time efficient, the metamodeling steps can be skipped. Note that the meta-model can only be used in the range of the training data values.

To balance between time efficiency and reliability, this paper proposes following strategy as also illustrated in Fig. 1 [29]. First, an extra sampling set is needed. One of the two sets is selected as training set to build the model. Then a $k$-fold cross-validation is performed to control the reliability with validation indicators, which indicate how well the original model is approximated. This means that each sample set is once used as validation set, while the other sets are training sets, resulting in as many validation indicator values as available sample sets (i.e. k). Among others coefficients of determination $r^{2}$, indicating the overall fit, and maximal errors can be used as indicators. These steps are repeated until the convergence of minimal, maximal and average values of selected validation indicators is satisfactory: adding more sample sets does not change the values more than a user defined percentage. Reliability of the meta-model is then judged based on the converged indicators. Which accuracy is needed is problem dependent. If the built meta-model remains unsufficiently reliable, another meta-modelling technique can be selected to obtain a potentially better reliability for the converged validation indicators.

In the example, a meta-model is required to replace the transient BES-model. Seven sample sets, thus six column permutations from the original maximin sampling scheme of 5.2.1, were needed to obtain a reliable MARS model. The average, minimal and maximal $r^{2}$ values vary about 1\%. As can be seen in Fig. 5, the meta-model approximates the original simulation model very well with $r^{2}$ equal to 0.97 for one validation set of 20 samples. The average, minimal and maximal $r^{2}$ in the cross-validation is respectively $0.95,0.93$ and 0.97 . For convergence of maximal errors, more sample sets are needed. After seven sets, the average, minimal and maximal maximal error in the crossvalidation is respectively 13,10 and $21 \mathrm{kWh} / \mathrm{m}^{2}$ and after ten sets 12,9 and $15 \mathrm{kWh} / \mathrm{m}^{2}$.

\subsubsection{Sensitivity analysis}

The previously run Monte Carlo sets are used for a sensitivity analysis to determine the most influencing parame- 


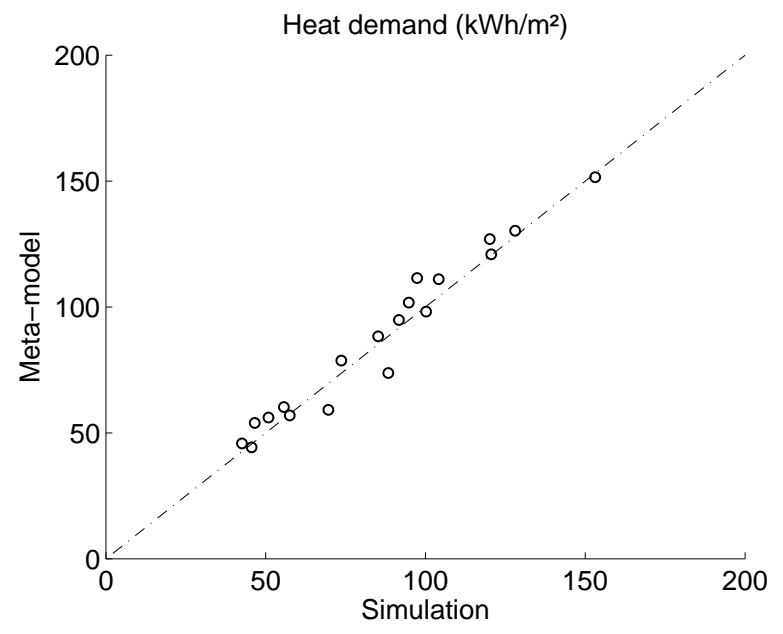

Figure 5: Comparison simulated and meta-modeled heat demand of validation data set.

ters. First, the results need to be post-processed to obtain the derived output parameters. The sensitivity indices can be calculated in several ways, such as Pearson productmoment correlation coefficient $r$ or Spearman's rank correlation coefficients $\rho$, and visualised with scatter plots [30]. The meta-model can provide some information on the influencing input parameters as well.

Since as few samples as needed are preferred in the screening phase, it is essential to check the significance of influencing parameters or the convergence of the indices. $\mathrm{P}$-values can be calculated for significance control by testing the hypothesis of no correlation. These p-values are the probability of randomly getting a correlation as large as calculated, when the true correlation is zero. This means that low p-values, usually smaller than 0.05 , indicate significant correlations. Typically, these p-values are larger for less influencing parameters and when less samples are used. If some parameters show significant impact on the output, one can conclude the preliminary screening; otherwise, more samples are needed. The convergence of the indices, on the other hand, can be controlled using the sampling sets needed for meta-modeling. The indices are sufficiently converged when adding sets to the sampling scheme does not change their values more than a user defined percentage, eg. $5 \%$. More samples can be added if the indices are not yet converged.

To illustrate this, the Spearman's rank correlations between the net present cost and the contributing input parameters are calculated, as well as their p-values. Table 2 shows the parameters in descending order of influence. As can be seen, increasing absolute $\rho$ means decreasing $\mathrm{p}$. In this example, only the nominal energy price evolution, the sunscreen type and the infiltration rate significantly influence the net present cost. The corresponding $\rho$-values are converged as well, as adding the final sample set changes the values less than $5 \%$.

As the net present cost is highly influenced by the nominal energy price evolution and this parameter will be con- sidered as scenario parameter in section 5.4, the sensitivity analysis is repeated for a constant energy price evolution (in this case $2.3 \%$ ) in Table 3. Again the sunscreen type and infiltration rate, but also the U-value of the wall, significantly influence the net present cost. To get the comprehensive picture, this can be analogously done for the other scenario values.

As a MARS model takes only most influencing param-

Table 2: Spearman's $\rho$ and p-values of net present cost in descending order of influence.

\begin{tabular}{|l|l|l|}
\hline PARAMETER & $\boldsymbol{\rho}$ & $\mathbf{P}$ \\
\hline Nominal energy price evolution & 0.76 & 0 \\
Sunscreen type & 0.39 & 0 \\
Infiltration rate at 50 Pa & -0.16 & 0.03 \\
Ventilation system & 0.12 & 0.09 \\
Heat recovery & 0.10 & 0.15 \\
U-value wall & -0.08 & 0.26 \\
Set temperature occupancy night & -0.06 & 0.38 \\
zone & & \\
U-value roof & -0.06 & 0.38 \\
Workmanship error U-values & 0.05 & 0.48 \\
Set temperature occupancy day & 0.05 & 0.50 \\
zone & & \\
Air change rate & -0.05 & 0.52 \\
U-value floor type & -0.04 & 0.58 \\
Window type & 0.04 & 0.60 \\
Set temperature absence day zone & -0.03 & 0.71 \\
Internal heat gains & -0.02 & 0.74 \\
Workmanship error infiltration & -0.02 & 0.78 \\
rate & & \\
\hline
\end{tabular}

Table 3: Spearman's $\rho$ and p-values of net present cost without scenario parameter in descending order of influence.

\begin{tabular}{|l|l|l|}
\hline PARAMETER & $\boldsymbol{\rho}$ & $\mathbf{P}$ \\
\hline Sunscreen type & 0.71 & 0 \\
Infiltration rate at 50 Pa & -0.23 & 0 \\
U-value wall & -0.16 & 0.03 \\
Set temperature occupancy day & 0.12 & 0.10 \\
zone & & \\
Heat recovery & 0.09 & 0.23 \\
Set temperature occupancy night & -0.08 & 0.25 \\
zone & & \\
Ventilation system & 0.08 & 0.28 \\
Set temperature absence day zone & -0.08 & 0.28 \\
Workmanship error U-values & 0.07 & 0.28 \\
Internal heat gains & -0.07 & 0.32 \\
Workmanship error infiltration & -0.07 & 0.32 \\
rate & & \\
U-value floor & -0.07 & 0.34 \\
Window type & -0.06 & 0.41 \\
Air change rate & 0.05 & 0.52 \\
U-value roof & -0.03 & 0.73 \\
\hline
\end{tabular}


eters into account, the created heat demand meta-model, replacing the original BES-model, can equally be used as sensitivity tool for the heat demand. The contributing parameters are indicated in the third column of Table 1. When calculating $\rho$ as well, these parameters are indeed most influencing for heat demand. As heat demand is only part of the net present cost, important parameters for heat demand might be less important for net present cost. Furthermore, decreasing energy cost due to design options might be associated with increasing investment costs, resulting in a zero contribution. However, the dominant parameters for net present cost of Table 3 also appear in the meta-model.

\subsection{Updating}

Based on the sensitivity results, one can judge which parameters need accurate distributions and which parameter variations can be neglected. Omitting input parameters with little influence reduces labour cost to find all distributions, and may improve sampling efficiency of space-filling sampling schemes. Moreover, this can limit the number of design options in the probabilistic design of section 5.4.1, significantly improving optimisation efficiency.

In this example, the U-values of roof and floor and both workmanship parameters are omitted as their variation was apparently not significant for the heat demand, nor for the net present value. The respective average values are therefore considered, as shown in Table 1. As the distributions of design, uncertainty and scenario parameters were already based on a measurement campaign, no further adjustments are needed. Based on the sensitivity results, one can expect that design parameters sunscreen type, infiltration rate, U-value of wall and perhaps heat recovery of the ventilation system will dominate the optimal design options.

When a sufficient number of measurements is available, the input distributions of the most influencing parameters can be refined based on Bayesian calibration [9, 10], which is however out of the scope of this paper.

\subsection{Probabilistic design}

After the problem is screened and all input distributions are known, the output distributions can be calculated in a Monte Carlo loop. Therefore, all considered design options are combined in a multi-layered sampling scheme with scenario options and uncertain parameters (see section 5.4.2). The Monte Carlo loop itself is explained in section 5.4.3. The output distributions allow comparing and optimising the effectiveness and robustness of design options, as defined in section 3 and illustrated in section 5.4.4.

\subsubsection{Design options}

Several possibilities are available to select the considered design options as included in Table 1. A full factorial scheme, as will be illustrated in this paper, is the simplest and thus default option. In such a scheme, discrete potential values - called levels - are selected for each design parameter - called factors - and all level combinations across all factors are made. In this way, a visual presentation of all potential design options is possible. The sensitivity results can be used to guide to the range of potential optimal levels. If numerous design options are considered, it is possible to study a coarse scheme with few levels or a fractional factorial scheme first and then to refine based on the corresponding results.

In the case study, every design parameter is sampled with several design values (see Table 1 column 4) and these values are combined in a full factorial scheme, leading to 640 considered design options in the design layer.

The design options can alternatively be selected based on an optimisation algorithm such as a genetic algorithm. A first guess is made for the optimal design and the design parameters are adjusted based on the calculated outputs. Unfortunately, some minor changes are needed in the output evaluation in this case as will be explained in section 5.4.4.

Note that in a probabilistic analysis, only one design is investigated, thus examined as a probabilistic design problem with only one considered design option.

\subsubsection{Multi-layered sampling scheme}

Besides the controllable design options, scenario and uncontrollable inherently uncertain parameters are part of the probabilistic design problem, as presented in Table 1, and are combined in a multi-layered sampling scheme as aleady shown in Fig. 2.

In the simplest case, the multi-layered sampling scheme consists of only two layers: the design options and the uncertain parameters. The values of the uncertainty layer parameters, as seen in Table 1 for the case study, are combined with all created design options, so that every design undergoes the same uncertainties. To create these uncertainty layer values, several sampling techniques can be used. As stated in 5.2, small replicated space-filling schemes are recommended.

If the explicit evaluation of the result for potential scenarios is requested, more layers can be added. In the case study, one is interested in the design option with the highest effectiveness and robustness for net present cost, regardless of the future economic situation. Therefore, the energy price evolution is considered in the scenario layer and not in the uncertainty layer. The latter could be the case if the explicit evaluation was not asked. Again, all design options are combined with all uncertainty layer values and all scenario layer values, which is in fact a full factorial scheme of the layer values. The scenario layer can be created with a small sampling scheme or based on potential discrete scenario options, such as in Table 1 for the example. In more advanced cases, more scenario layers can be taken into account as well. 


\subsubsection{Monte Carlo loop}

All value combinations of the multi-layered scheme, as presented in Fig. 2, are run in a Monte Carlo loop while checking convergence for the outputs. To obtain reliable results, all considered output indicators should converge. In this paper, we are interested in the minimum, the median and the $2.5 \%$ and $97.5 \%$ percentiles of the net present cost to check for the cost-robust and cost-effective solutions as explained in section 3. These indicators are sufficiently converged when adding samples to the sampling scheme, does not change their values more than a user defined percentage, for instance $5 \%$.

First, a single design and scenario value are selected and the uncertainty layer values are run, as seen in Fig. 1. This layer scheme is enlarged until the output indicators reach convergence. Secondly, the following scenario value is selected and again the uncertainty layer is run and enlarged until convergence. This is analogously done for all other scenario values. Thereafter, the convergence of the scenario layer is investigated and if necessary, more scenario samples can be added. The latter is not needed when discrete, equiprobable scenarios are used in the scenario layer. After all output indicators converge on every layer, one can continue with the next design option. If more scenario layers are taken into account, this Monte Carlo loop can be easily expanded.

In the considered case study, all design options are run under influence of the scenario and uncertainty layer, resulting in 640 cumulative distribution functions for net present cost as shown in Fig. 6. The first design option is indicated in red, and this function is in fact the result of a probabilistic analysis for that option. The vertical shift at $67 \%$ is due to the third considered economical scenario. An energy price evolution of $10 \%$ will highly increase the net present cost.

To illustrate the convergence principle, the convergence of the first design option is given. For the three scenarios,

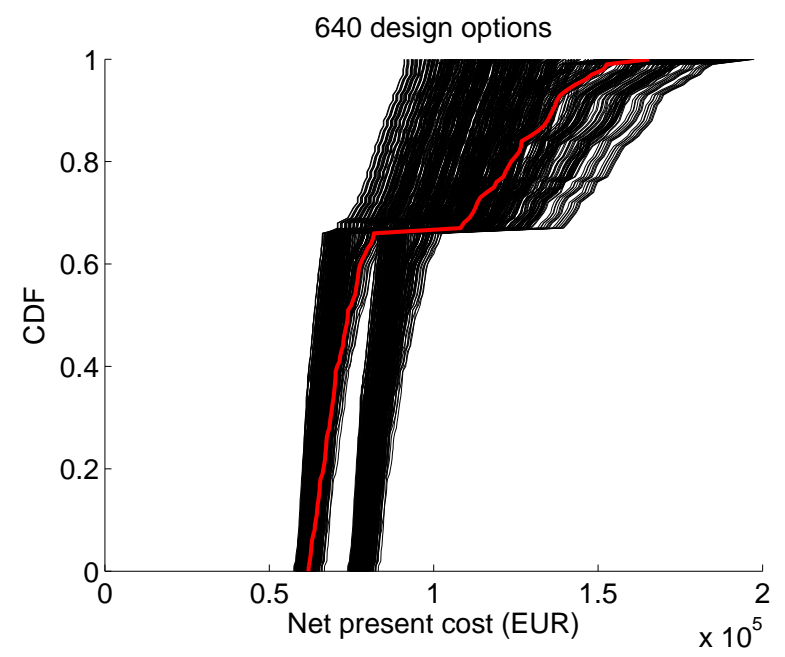

Figure 6: Cumulative distribution functions of net present cost for all design options. First design option is indicated in red. after two uncertainty layer sets, the $2.5 \%, 50 \%$ and $97.5 \%$ percentiles vary less than $0.73 \%$, which is already sufficiently converged. After five sets, the values vary even less than $0.04 \%$. As five sets of 20 samples were calculated for all three scenario values of the design option to illustrate this, the cumulative distribution is created from 300 runs.

\subsubsection{Output evaluation}

All outputs calculated, the cumulative distribution functions allow a visual selection of the optimal designs (i.e. the cumulative distribution function the most to the left in Fig. 6 for the current case study, as this has the lowest median and spread in net present cost), but when numerous design options and multiple output parameters are included, this becomes less feasible. Therefore, median values and percentile differences can be used to numerically evaluate them [31]. To use these values in optimisation, effectiveness $\varepsilon$ and robustness $R_{P}$ were proposed as output uncertainty indicators in section 3 .

If a full factorial scheme is applied for the design options, $\varepsilon$ and $R_{P}$ can be easily calculated for all design options and scenarios because all percentiles are known. For convergence reasons design and scenario options might have respectively scenario and uncertainty layers with different sizes. Since this influences the overall and design percentiles in Eq. 1 and 2, weight factors need to be used to equally weigh every branch in the multi-layered scheme.

As an illustration, Fig. 7 shows robustness $R_{P}$ of the net present cost in function of effectiveness $\varepsilon$ for all design options, subjected to both uncertainty and scenario parameters. The Pareto optimal design options can be calculated. These options include the lower infiltration rates, a balanced ventilation system, the lower U-values for walls and windows and no sunscreens. Because of the economic scenario layer in the multi-layered sampling scheme, it is advised to check these overall optimal designs for potential economical scenarios, which is done in Fig. 8. $\varepsilon$ and $R_{P}$ are therefore calculated per scenario option. One can see that the selected optimal designs are not really optimal in all scenarios, but close to optimal, indicating that they are reliable in this case study. One can see that the variability of the net present cost is reduced while selecting the scenario values and thus that the $R_{P}$ indicators are increased, as the energy price evolution is highly influencing the net present cost. The difference in robustness between the design options is therefore also reduced. When looking at the effectiveness of the design options, one can see that this can be highly influenced by the future scenario as well. Selecting one of the overall Pareto front solutions is in this case advised.

Note that, when an optimisation algorithm is used for the design options instead of a full factorial design, only the design option percentiles are known in Eq. 1 and 2 . Therefore, the overall percentiles in the denominator are yet to be calculated with a new design layer scheme which is preferably space-filling sampled. This layer is also enlarged until convergence is reached for the used percentiles. 


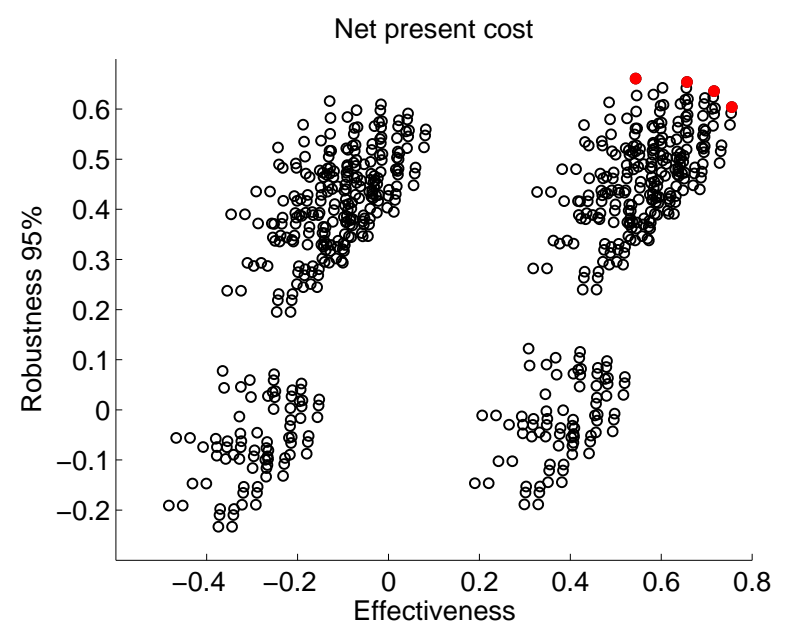

Figure 7: Robustness of net present cost in function of effectiveness for all design options. Pareto front is indicated in red.

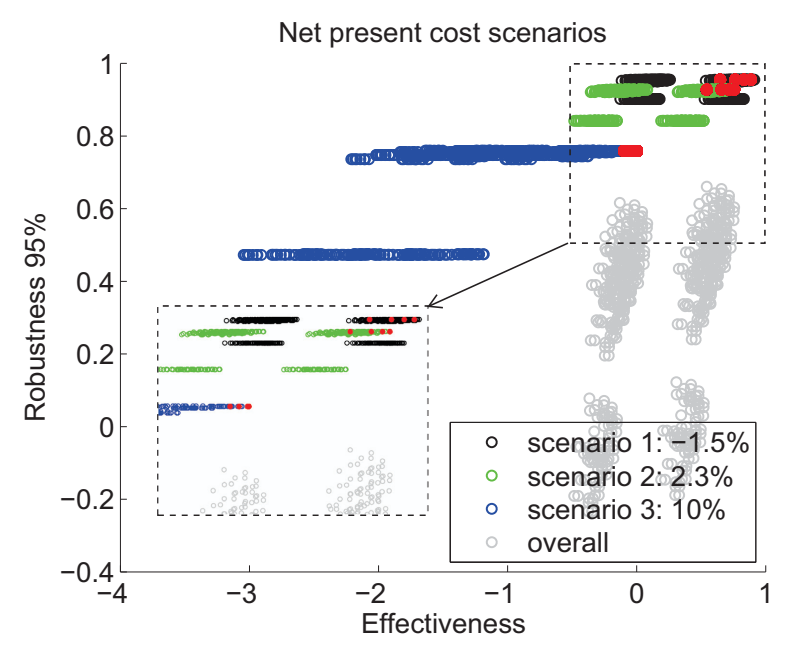

Figure 8: Robustness of net present cost in function of effectiveness for all design options for three scenarios. Overall robustnesses and effectivenesses of Fig. 7 are indicated in grey. Overall optimal designs of Fig. 7 are indicated in red. A snapshot of the Pareto front is shown as well.

If no scenario layers are considered, the preliminary screening can provide the overall percentiles.

\section{Discussion}

Section 5 described and illustrated the methodology as introduced in section 3 . Note that the example is greatly simplified to clearly illustrate the methodology. To omit additional conceptual difficulties, not all contributing input and output parameters were considered. In further research, the methodology will be applied on more realistic case studies.

In this example, the problem was first screened to reduce the number of considered input parameters and to improve calculation efficiency using meta-models. Then, a multi-layered sampling scheme, containing all design, scenario and inherently uncertain parameters, was run in a
Monte-Carlo loop. To facilitate numerical optimisation of the probabilistic outputs, effectiveness and robustness indicators of the design options were calculated.

Instead of using Pareto fronts, as illustrated in Fig. 7, the weighted sum method can also be used to optimise multiple effectiveness and robustness indicators. This enables to attach more importance to some performance parameters or to only effectiveness or robustness.

In more advanced applications, it is conceivable that not all output parameters are considered based on their distribution. Designs resulting in overheating problems, for example, can easily be penalised or even be excluded based on indoor temperatures. In the weighted sum method, also large weight factors can be chosen to minimise these undesired performances.

As shown here, the proposed methodology is widely applicable and moreover many issues, such as sampling efficiency, output convergence and calculation time, have been tackled. However, two challenging issues still need to be investigated in further research.

First of all, more attention is needed on input distributions. As collecting very detailed distributions and correlations might be very time consuming or even unfeasible and simplified distributions may cause wrong results, more knowledge is crucial to determine how accurate the distributions and correlations should be. In some cases, simple input ranges might satisfy, while in others more detailed information is needed. At the same time, correlations between the input parameters might exist (eg. U-value and air tightness) and therefore may have a significant influence as well, but this is hard to determine.

Secondly, to obtain reliable optimisation results, the meta-model needs to be sufficiently accurate. Therefore, meta-models are validated and cross-validated with validation criteria handling the overall fit and maximal error as explained in section 5.2.2. As accuracy might be case dependent, the validation criteria has to be studied for several cases to optionally generalise them.

\section{Conclusions}

To analyse building performances and to take reliable design decisions while including input uncertainties, this paper proposed a probabilistic methodology. In this methodology, probabilistic analysis is seen as a special design case. As seen in Fig. 1, the methodology consists of four main steps:
1. Preprocessing
2. Preliminary screening
3. Updating
4. Probabilistic design

In the preprocessing step a simulation model and input and output parameters are selected. All input parameters are assigned fixed values or probability distributions as accurate as known for the considered problem. Each 
parameter can be variable in time and space as well and correlations can be taken into account.

The preliminary screening constructed a meta-model to replace the original model for time-inefficient models. Then the input parameters samples are used to calculate sensitivity indices. Both sampling efficiency and output convergence are therefore considered.

These sensitivity indices are used to update the input parameters. Based on the ranking, one can judge which parameters need accurate distributions and which can be neglected to improve design and sampling efficiency.

To subject all design options to the same uncertainties and moreover to check the validity of optimisation results for potential scenarios, a multi-layered sampling scheme is created in the actual probabilistic design step. This scheme contains the design options, the scenarios and the inherently uncertain parameters. All values of this scheme are combined across the layers and run in a Monte-Carlo loop while controlling output convergence.

To facilitate numerical evaluation and optimisation of the output distributions, effectiveness and robustness indicators were proposed, inspired by robust design. Effectiveness is defined as the ability of the design option to optimise the performance, while robustness is defined as the ability to stabilise this performance for the entire range of input uncertainties.

This methodology outlines the steps needed to be taken in both reliable probabilistic analysis and design, making many applications in research, industry and government feasible.

\section{Acknowledgements}

The authors are very thankful for the fundings of the Flemish government and companies for the IWT TETRA BEP2020 project and participating inhabitants for the related measurement campaign. Many thank to Liesbeth Staepels and PHL University College / Hasselt University to develop a cost calculation tool according to the European standard EN ISO 15459 [22] in the framework of this research project [23].

They would like to thank Bart Husslage and Gijs Rennen from the Tilburg University as well for sharing their MATLAB code for calculation of maximin sampling schemes [27].

\section{References}

[1] M. Abuku, H. Janssen, S. Roels, Impact of wind-driven rain on historic brick wall buildings in a moderately cold and humid climate: Numerical analyses of mould growth risk, indoor climate and energy consumption, Energy and Buildings 41 (1) (2009) 101-110.

[2] G. Verbeeck, H. Hens, Life Cycle Optimization of Extremely Low Energy Dwellings, Journal of Building Physics 31 (2) (2007) 143-177.

[3] B. Sudret, Uncertainty propagation and sensitivity analysis in mechanical models: Contributions to structural reliability and stochastic spectral methods, 2007.
[4] K. J. Lomas, H. Eppel, Sensitivity analysis techniques for building thermal simulation programs, Energy and Buildings 19 (1) (1992) 21-44.

[5] I. A. Macdonald, P. Strachan, Practical application of uncertainty analysis, Energy and Buildings 33 (3) (2001) 219-227.

[6] H. Janssen, Monte-Carlo based uncertainty analysis: Sampling efficiency and sampling convergence, Reliability Engineering \& System Safety 109 (2013) 123-132.

[7] S. Burhenne, O. Tsvetkova, D. Jacob, G. P. Henze, A. Wagner, Uncertainty quantification for combined building performance and cost-benefit analyses, Building and Environment 62 (2013) $143-154$.

[8] B. Eisenhower, Z. ONeill, S. Narayanan, V. a. Fonoberov, I. Mezić, A methodology for meta-model based optimization in building energy models, Energy and Buildings 47 (2012) 292301.

[9] A. Booth, R. Choudhary, D. Spiegelhalter, Handling Uncertainty in Housing Stock Models, Building and Environment 48 (2012) 35-47.

[10] A. Booth, R. Choudhary, Decision making under uncertainty in the retrofit analysis of the UK housing stock: Implications for the Green Deal, Energy and Buildings 64 (2013) 292-308.

[11] C. Zang, M. Friswell, J. Mottershead, A review of robust optimal design and its application in dynamics, Computers \& Structures 83 (4-5) (2005) 315-326.

[12] S. M. Sanchez, Robust design: seeking the best of all possible worlds, in: J. A. Joines, R. R. Barton, K. Kang, P. A. Fishwick (Eds.), Proceedings of the 2000 Winter Simulation Conference, 2000.

[13] P. Hoes, M. Trcka, J. L. M. Hensen, B. Hoekstra Bonnema, Optimizing building designs using a robustness indicator with respect to user behavior, in: Proceedings of the 12th Int. IBPSA conference, Sydney, 14-16 November, Sydney, 1710-1717, 2011.

[14] S. M. Sanchez, P. J. Sanchez, J. S. Ramberg, F. Moeeni, Effective engineering design through simulation, International Transactions in Operational Research 3 (2) (1996) 169-185.

[15] C. J. Hopfe, J. L. Hensen, Uncertainty analysis in building performance simulation for design support, Energy and Buildings 43 (10) (2011) 2798-2805.

[16] H. van den Berg, Robust design of structures using uncertainty methods combined with Component Mode Synthesis, Ph.D. thesis, Technische Universiteit Eindhoven, 2005.

[17] C. J. Hopfe, M. T. M. Emmerich, R. Marijt, J. Hensen, Robust multi-criteria design optimisation in building design, in: First Building Simulation and Optimization Conference, 10-11 September 2012, Loughborough, UK, 19-26, 2012.

[18] C. J. Hopfe, G. Augenbroe, J. L. Hensen, Multi-Criteria Decision Making Under Uncertainty In Building Performance Assessment, Building and Environment 69 (2013) 81-90.

[19] L. Van Gelder, H. Janssen, S. Roels, G. Verbeeck, L. Staepels, Effective and robust measures for energy efficient dwellings: probabilistic determination, in: Proceedings of 13th International Conference of the International Building Performance Simulation Association, August 25-28 2013, France, 2013a.

[20] R. Baetens, R. De Coninck, J. Van Roy, B. Verbruggen, J. Driesen, L. Helsen, D. Saelens, Assessing electrical bottlenecks at feeder level for residential net zero-energy buildings by integrated system simulation, Applied Energy 96 (2012) 74-83.

[21] EPBD, Directive 2002/91/EC of the European Parliament and of the Council of 16 December 2002 on the energy performance of buildings .

[22] EN ISO 15459, Energy performance of buildings - Economic evaluation procedure for energy systems in buildings .

[23] G. Verbeeck, L. Staepels, S. Roels, L. Van Gelder, G. Bauwens, A.-H. Deconinck, IWT TETRA BEP2020: betrouwbare energieprestaties van woningen - Naar een robuuste en gebruikersonafhankelijke performantie (in Dutch), URL http://bep2020.pxl.be/, 2013.

[24] NBN D 50-001, Ventilatievoorzieningen in woongebouwen (in Dutch).

[25] O. Guerra Santin, L. Itard, H. Visscher, The effect of occupancy 
and building characteristics on energy use for space and water heating in Dutch residential stock, Energy and Buildings 41 (11) (2009) 1223-1232.

[26] L. Staepels, G. Verbeeck, S. Roels, L. Van Gelder, G. Bauwens, Do Ventilation Systems Accomplish the Necessary Indoor Air Quality in Low Energy Houses?, in: CLIMA 2013: June 16-19, Prague, 2013.

[27] B. Husslage, G. Rennen, E. R. van Dam, D. den Hertog, Spacefilling Latin hypercube designs for computer experiments, 2008.

[28] J. H. Friedman, Multivariate adaptive regression splines, The Annals of Statistics 19 (1991) 1-141.

29] L. Van Gelder, H. Janssen, S. Roels, Metamodelling in robust low-energy dwelling design, in: Proceedings of 2nd Central European Symposium on Building Physics, September 9-11 2013, Vienna, 2013b.

[30] D. M. Hamby, A review of techniques for parameter sensitivity analysis of environmental models, Environmental Monitoring and Assessment 32 (2) (1994) 135-154.

31] V. Rathod, O. P. Yadav, A. Rathore, R. Jain, Optimizing reliability-based robust design model using multi-objective genetic algorithm, Computers \& Industrial Engineering 66 (2) (2013) 301-310. 